Luiz Souza lima da Silva Carvalho

\title{
A MINERAÇÃO RESPONSÁVEL: \\ LIMITES PARA A AUTORREGULAÇÃO SOCIOAMBIENTAL DO SETOR MINERAL NO BRASIL
}

TESE DE DOUTORAMENTo

Orientadora: Professora Associada ANa MARIa de Oliveira NuSDEO

Faculdade de Direito da Universidade de São Paulo

São Paulo - 2014 


\title{
A MINERAÇÃO RESPONSÁVEL:
}

\section{LIMITES PARA A AUTORREGULAÇÃO SOCIOAMBIENTAL DO SETOR MINERAL NO BRASIL}

\author{
Tese de Doutoramento em Direito \\ Ambiental apresentada ao Departamento de \\ Direito Econômico, Financeiro e Tributário, \\ sob orientação da Professora Associada \\ Ana Maria de Oliveira Nusdeo.
}

Faculdade de Direito da Universidade de São Paulo

São Paulo, 2014 


\section{FOLHA DE APROVAÇÃO}

Luiz Souza Lima da Silva Carvalho

A Mineração Responsável: limites para a autorregulação socioambiental do setor mineral no Brasil

Tese de Doutoramento apresentada ao Departamento de Direito Econômico, Financeiro e Tributário para obtenção do título de Doutor em Direito Ambiental

Aprovado em:

Banca Examinadora:

Prof. Dr.

Instituição

Assinatura:

Prof. Dr.

Instituição Assinatura:

Prof. Dr.

Instituição Assinatura:

Prof. Dr.

Instituição Assinatura:

Prof. Dr. Instituição Assinatura: 


\section{DEDICATÓRIA}

Ao Francisco, pois os filhos são a prova mais contundente da existência de Deus.

\section{AGRADECIMENTOS}

Agradeço à minha esposa, Daniela, e à minha família, pelo amor e apoio a todo o instante. Agradeço ao meu sócio, Marco, e à minha equipe do BCA pela compreensão e auxílio durante esse longo período.

Agradeço à minha orientadora, Professora Ana Maria, pela paciência e atenção.

Agradeço à Carol, pelo auxílio incansável para que a pesquisa fosse adiante.

Agradeço aos Srs. Antero Saraiva Junior e Antonio Camargo Junior pela disponibilidade e atenção dispensadas para a formatação do questionário para as partes interessadas.

Agradeço a todos que, mesmo com a agenda apertada, puderam responder ao questionário, enriquecendo essa pesquisa.

Agradeço aos professores Alessandro Octaviani, Diogo Coutinho, Fernando Scaff e Gilberto Bercovici pelas críticas e pelos direcionamentos. 


\section{LISTA DE SIGLAS E ABREVIATURAS}

ABNT Associação Brasileira de Normas Técnicas

AF\&PA American Forest \& Paper Association Sustainable Forest Initiative

AMN Associação Mercosul de Normalização

ANA Agência Nacional das Águas

ASI Acreditation Service International

BSCI Business Social Compliance Initiative

CAB Organismo de Avalição de Conformidade (Conformity Assesment Body)

Cadmadeira Cadastro Estadual das Pessoas Jurídicas que comercializam, no Estado de São Paulo, produtos e subprodutos florestais de origem nativa da flora brasileira

CBPM Companhia Baiana de Pesquisa Mineral

CECAV Centro de Estudos de Cavernas

CERFLOR Programa Brasileiro de Certificação Florestal

CETEM Centro de Tecnologia Mineral

CETESB Companhia de Tecnologia de Saneamento Ambiental

CFEM Compensação Financeira pela Exploração dos Recursos Minerais

CIES Comite' International d'Entreprises a` Succursales

CNRH Conselho Nacional de Recursos Hídricos

CONAMA Conselho Nacional de Meio Ambiente

COPANT Comissão Pan-americana de Normas Técnicas

CORI Comitê Orientador para Implementação da Logística Reversa 
CPRM Serviço Geológico Brasileiro - CPRM

CSA Canadian Standards Association Group

DNPM Departamento Nacional de Produção Mineral

EIA Estudo Prévio de Impacto Ambiental

FSC Forest Stewardship Council

FTA Foreign Trade Association

FUNAI Fundação Nacional do Índio

IBAMA Instituto Brasileiro de Meio Ambiente e Recursos Naturais Renováveis

ICEM International Federation of Chemical, Energy, Mine and General Workers Unions

ICME International Council on Metals and the Environment

ICMI International Cyanide Management Institute

ICMM International Council on Mining and Metals

IEC International Eletrotechnical Comission

IFC International Finance Coorporantion

IIED International Institute for Environment Development

IRMA Initiative for Responsible Mining Assurance

ISEAL Aliança Internacional de Rotulagem e Acreditação Social e Ambiental

ISO International Organization for Standardization

LEED Leadership in Energy and Environmental Design

LI Licença de Instalação

LO Licença de Operação 
LP Licença Prévia

MCEP Mining Certification Evaluation Project

MDIC Ministério do Desenvolvimento, Indústria e Comércio Exterior

MMA Ministério do Meio Ambiente, dos Recursos Hídricos e da Amazônia Legal

MME $\quad$ Ministério de Minas e Energia

NSMD Non-state Market Driven

OMC Organização Mundial do Comércio

ONU Organização das Nações Unidas

OIT Organização Internacional do Trabalho

OMC Organização Mundial do Comércio

PAE Plano de Aproveitamento Econômico

PCA Plano de Controle Ambiental

PEFC Pan European Forest Certification Council

PMQA Programa Municipal de Qualidade Ambiental

PMSP Prefeitura Municipal de São Paulo

PNRS Política Nacional dos Resíduos Sólidos

PRAD Plano de Recuperação de Áreas Degradadas

RCA Relatório de Controle Ambiental

RIMA Relatório de Impacto Ambiental

SFI Sustainable Forestry Initiative

SGM Secretaria de Geologia, Mineração e Transformação Mineral 
SISNAMA Sistema Nacional de Meio Ambiente

SMA Secretaria do Meio Ambiente do Estado de São Paulo

UC Unidade de Conservação

UNCTAD UN Conference on Trade and Development

WBCSD World Business Council for Sustainable Development

WWF World Wide Fund for Nature 


\section{LISTA DE TABELAS}

Tabela 1 - Regulação minerária e ambiental da mineração

Tabela 2 - Princípios para uma mineração responsável do ICMM

Tabela 3 - Principais atos normativos de regulação socioambiental da mineração vigentes

Tabela 4 - Entidades mapeadas como relevantes para a certificação socioambiental da mineração de agregados para a construção civil no Estado de São Paulo

Tabela 5 - Princípios ICMM

Tabela 6 - Impactos socioambientais da mineração de areia

Tabela 7 - Impactos socioambientais da mineração de rocha

Tabela 8 - Entidades mapeadas por setores para aplicação de questionário 


\section{LISTA DE FIGURAS}

Figura 1 - Pirâmide de estímulos e sanções

Figura 2 - Modelo geral de sistema de certificação

Figura 3 - Gráfico demonstrativo dos stakeholders do setor minerário

Figura 4 - Recorte das primeiras décadas de atuação da Cetesb acerca da mineração e meio ambiente

Figura 5 - Maiores empresas de mineração do Estado de São Paulo

Figura 6 - Participação no consumo de areia

Figura 7 - Estrutura do sistema de certificação socioambiental da mineração de agregados para a construção civil do Estado de São Paulo

Figura 8 - Principais desafios apontados pelas partes interessadas

Figura 9 - Principais desafios apontados pelos entrevistados de acordo com o setor de atuação

Figura 10 - Comparação dos desafios apontados pelas partes interessadas entrevistadas com os princípios do ICMM

Figura 11 - Mapeamento de partes interessadas indicadas pelos entrevistados segundo o setor de atuação

Figura 12 - Recorrência de citação de partes interessadas pelos entrevistados 


\section{LISTA DE GRÁFICOS}

Gráfico 1 - Mercados estaduais de agregados para a construção civil

Gráfico 2 - Apoio das partes interessadas à certificação socioambiental da mineração

Gráfico 3 - Comparação do apoio integral e do apoio com ressalvas dos entrevistados à certificação da mineração

Gráfico 4 - Participação dos entrevistados no sistema de certificação

Gráfico 5 - Comparação dos entrevistados que apoiariam com os que não apoiariam a iniciativa

Gráfico 6 - Percepção dos entrevistados acerca da pertinência da participação do Poder Público na iniciativa

Gráfico 7 - Ranking geral

Gráfico 8 - Ranking setorial 


\section{RESUMO}

O presente trabalho, intitulado A Mineração Responsável: limites para a autorregulação socioambiental do setor mineral no Brasil, teve por problema de pesquisa a possiblidade de estruturação e utilização da certificação socioambiental de terceira parte para a melhoria do desempenho ambiental das empresas de mineração de agregados no Estado de São Paulo. O problema de pesquisa foi formulado como questão de pesquisa com a seguinte pergunta: um sistema de autorregulação ambiental pode ser estruturado como um fator de melhoria na qualidade ambiental do setor minerário no Brasil? Frente a esta questão, foi tomada como hipótese de pesquisa a viabilidade do emprego da autorregulação socioambiental do setor mineral brasileiro, na forma de um sistema de certificação, desde que, entre outros fatores, essa escolha: 1) respeite as balizas constitucionais e o espaço não resguardado à regulação do Estado; 2) tenha princípios e diretrizes adequados ao contexto nacional; 3) respeite a uma lógica de governança legitimada; 4) haja posicionamento favorável das partes interessadas; e 5) haja a colaboração do Estado para a valorização da iniciativa.

Palavras-Chave: mineração, autorregulação, certificação, governança, legitimidade, partes interessadas. 


\begin{abstract}
This paper, entitled Responsible Mining: limits for social and environmental selfregulation of the mineral sector in Brazil, saw as a research problem the possibility of structuring and using third party environmental certification for improving the environmental performance of aggregate mining companies in the State of São Paulo. The research problem was drafted as a research question with the following question: Can an environmental self-regulation system be structured as a factor for improving environmental quality of the mining industry in Brazil? Faced with this question, the research hypothesis considered was the viability of using social and environmental self-regulation in the Brazilian mineral sector, in the form of a certification system, provided that, among other factors, this choice: 1) respects the constitutional references and the space not sheltered to State regulation; 2) has appropriate principles and guidelines to the national context; 3) respects the logic of legitimate governance; 4) has a favorable positioning by stakeholder; and 5) has the collaboration of the State for the development of the initiative.
\end{abstract}

Keywords: mining, self-regulation, certification, governance, legitimacy stakeholders. 


\section{RIASSUNTO}

Il presente lavoro, intitolato L'Attività Mineraria Responsabile: limiti per l'autoregolamentazione socio-ambientale del settore minerale in Brasile, ha scelto come problema di ricerca la possibilità di strutturazione e utilizzazione della certificazione socioambientale di terza parte per il miglioramento dello svolgimento ambientale delle aziende di attività mineraria di aggregati nello Stato di São Paulo. Il problema di ricerca è stato formulato come questione di ricerca con la seguente domanda: un sistema di autoregolamentazione ambientale può essere strutturato come un fattore di miglioramento nella qualità ambientale del settore minerario in Brasile? Davanti a tale questione, è stata presa come ipotesi di ricerca l'attuabilità dell'utilizzazione dell'autoregolamentazione socio-ambientale del settore minerale brasiliano nella forma di un sistema di certificazione, a condizione che, tra altri fattori, tale scelta: 1) rispetti i riferimenti costituzionali e lo spazio non osservato alla regolamentazione dello Stato; 2) abbia principi e direttive adeguati al contesto nazionale; 3) rispetti una logica di direzione legittimata; 4) abbia posizionamento favorevole delle parti interessate; e 5) abbia la collaborazione dello Stato per la valorizzazione dell'iniziativa.

Parole-chiave: attività mineraria, autoregolamentazione, certificazione, direzione, legittimità, parti interessate. 